\title{
Near-Field HF Antenna Pattern Measurement with Absolute Gain Calibration
}

\author{
Anirudha Siripuram* and Michael Daly \\ Applied Electromagnetics Branch, Code 52250 \\ SPAWAR Systems Center Pacific \\ San Diego, CA, USA \\ anirudha.siripuram@navy.mil, michael.p.daly@navy.mil
}

\begin{abstract}
A measurement method developed to extract the 3D antenna gain patterns of $\mathrm{HF}$ antennas is presented. The method employs a full hemispheric field probe measurement of an $\mathrm{HF}$ antenna on an outdoor antenna pattern range. A calibration method involving both simulation and measurement is used to transform antenna probe measurements into electric field quantities. The subsequent near-field electric field is extrapolated into the far-field using an established near-to-far-field transformation method.
\end{abstract}

Keywords—pattern measurement; near-field; HF antenna

\section{INTRODUCTION}

Antenna gain pattern measurement or verification is a critical step in an antenna's design cycle. In the case of HF $(2 \mathrm{MHz}$ to $30 \mathrm{MHz})$ antennas, measurement of such antennas not only requires a background environment that emulates the intended operational environment, but also affords an accurate means of collecting the necessary field data used to compute far-field quantities such as antenna gain. The Antenna Pattern Range (APR) maintained by SSC Pacific is proposed as means of satisfying the two requirements.

\section{APR MEASUREMENT}

\section{A. Physical Description of APR}

The APR consists of a fiberglass tripod arch on top of a conductive ground plane. The ground plane consists of a $12 \mathrm{ft}$ radius brass-plated turntable centered in an $80 \mathrm{ft}$ radius of $10 \mathrm{mil}$ aluminum on top of zinc. The fiberglass arch contains a track upon which a probe antenna sweeps through elevation angles between $3^{\circ}$ and $90^{\circ}$ while maintaining a radial distance of $80 \mathrm{ft}$ between the antenna and the center of the turntable. An antenna under test (AUT) can be placed in the center of the turntable and network analyzer "thru" measurements between the AUT and the probe antenna can be made for any desired combination of azimuth and elevation angles.

\section{B. Near-field Measurement}

Extraction of antenna gain patterns at HF requires a nearfield measurement. A thru measurement between the AUT and the probe is a scaled representation of the field at the location of the probe antenna. A calibration antenna can be measured to determine the value of the scale factor. A $35^{\prime}$ monopole and a $86 "$ dipole are chosen as the calibration and probe antennas respectively. A diagram of the measurement setup is shown in Fig. 1.

\footnotetext{
This work is supported by the Office of Naval Research Code 30 .
}

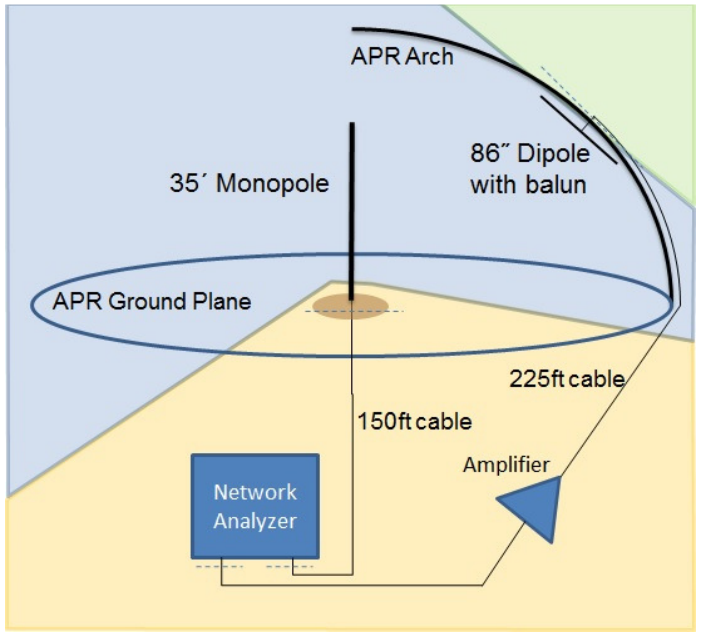

Figure 1: Measurement setup (35 ft. monopole as AUT)

The electromagnetic simulation software, FEKO [1], is used to model the calibration antenna and the APR facility such that a computed field value at a given measurement point can be used to relate an $S$-parameter thru measurement to a field quantity. A relationship between the near-field electric field emitted by an AUT (LHS term), the thru measurement for the AUT (numerator in RHS), the thru measurement for the calibration antenna at a calibration point (denominator in RHS), and the computed electric field at the given calibration point (first term in RHS) is given for the $\theta$-polarization in Eq. 1. A $\theta$-directed calibration polarization is also used in a similar $\varphi$-polarization equation not shown here. Once the tangential electric field emitted by an AUT is known at all measurement points on the APR hemisphere, the Equivalence Theorem and the alternative spherical harmonic expansion method of Sarkar et al. is used to extrapolate the near-field measurement into the far-field [2][3].

$$
E_{\theta}^{A U T}(\varphi, \theta)=E_{\theta}^{\text {Cal }, \text { sim }} \frac{S_{\text {THRU, }, \theta}^{A U T, \text { meas }}(\varphi, \theta)}{S_{\text {THRU }, \theta}^{\text {Cal,meas }}}
$$

\section{ERROR ESTIMATION}

Any evaluation of a measurement method requires some estimate of the method's error. A simple way to estimate the error is to first decompose Eq. 1 into its constituent parts as in Eq. 2. 


$$
E_{\theta}^{A U T}(\varphi, \theta)=S_{T H R U, \theta}^{A U T, \text { meas }}(\varphi, \theta) \frac{E_{\theta}^{\text {Cal,sim }}}{S_{\text {THRU }, \theta}^{\text {Cal,sim }}} \frac{S_{\text {THRU }, \theta}^{\text {Cal,sim }}}{S_{T H R U, \theta}^{\text {Cal,meas }}}
$$

The first term on the RHS is network analyzer measurement. Although there are errors associated with this term, it will be assumed that sufficient averaging, time-gating, and signal amplification are done such that the measurement error is negligible. The middle term in the RHS is the calibration term. The error that this term introduces is predominantly associated with the use of a single calibration point. The error associated with last term in the RHS is a consequence of the inaccuracies in the simulation model.

\section{A. Calibration Error}

A single calibration point was used $\left(0^{\circ} \mathrm{az}, 45^{\circ} \mathrm{el}\right)$ to simplify the measurement process. To estimate the subsequent calibration error, a simulation of the calibration and probe antennas can be used to compute the calibration term at some representative elevation angles. Comparison between the calibration terms at the representative elevation angles to the $45^{\circ}$ value can provide a means of estimating its error. The magnitude and phase errors are $[-4 \%$ to $1 \%]$ and $\left[-0.5^{\circ}\right.$ to $\left.1.5^{\circ}\right]$ respectively.

\section{B. Simulation Error}

One simple way to estimate the simulation error is to change the properties of a significant environmental parameter, such as the ground plane conductivity, and then observe the effect of the change in the simulated thru measurement. Such a comparison between the three representative elevation angles for the case of a realistic APR ground plane and a lossless ground is shown in Fig. 2, with magnitude and phase errors of $\pm 2 \%$ and $\pm 3^{\circ}$ respectively.
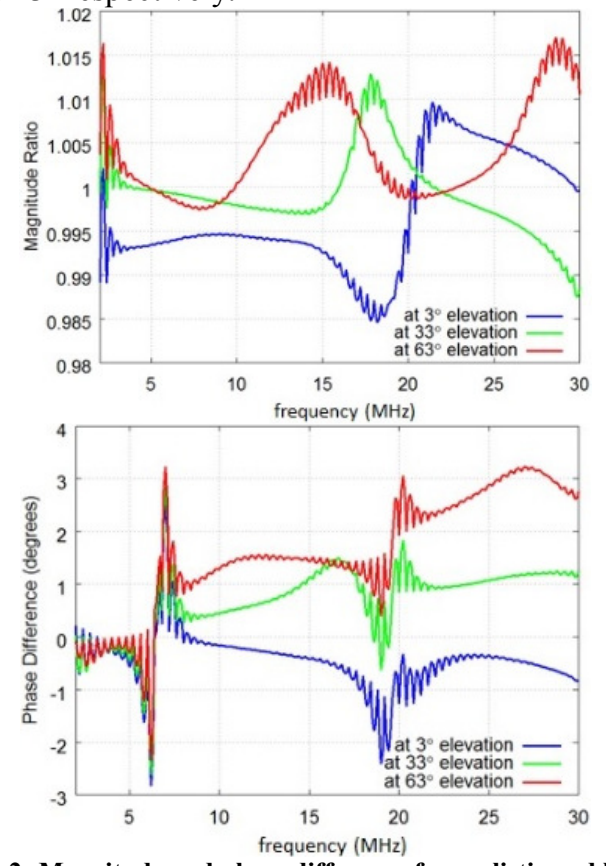

Figure 2: Magnitude and phase difference for realistic and lossless ground

\section{Overall Error Estimate \& Measurement}

Propagating the two errors through the near-to-far-field extrapolation using a simulated vertical Hertzian electric dipole source as an AUT reveals far-field errors within $\pm 5 \%$ (translates to $\pm 0.5 \mathrm{~dB}$ in gain error). To validate the error estimate from simulation, a dipole-loop antenna shown in Fig. 3 was both measured and simulated. The simulated vs. measured (adjusted for calibrated gain) field results in Fig. 4 are at $14 \mathrm{MHz}$.

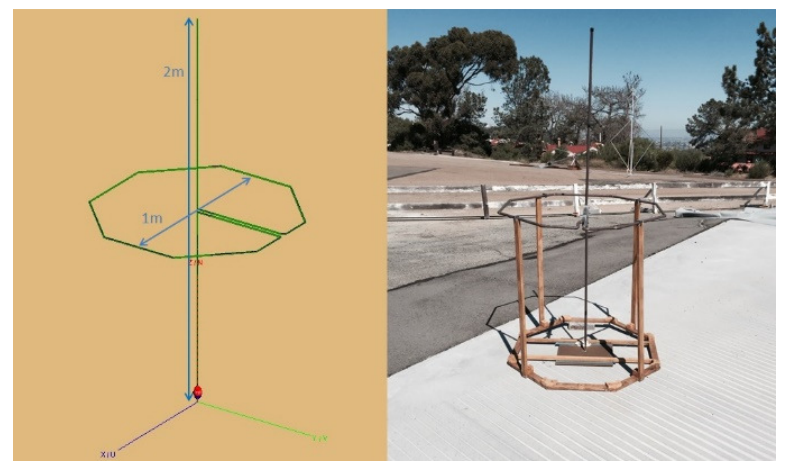

Figure 3: Dipole-loop antenna (example AUT)
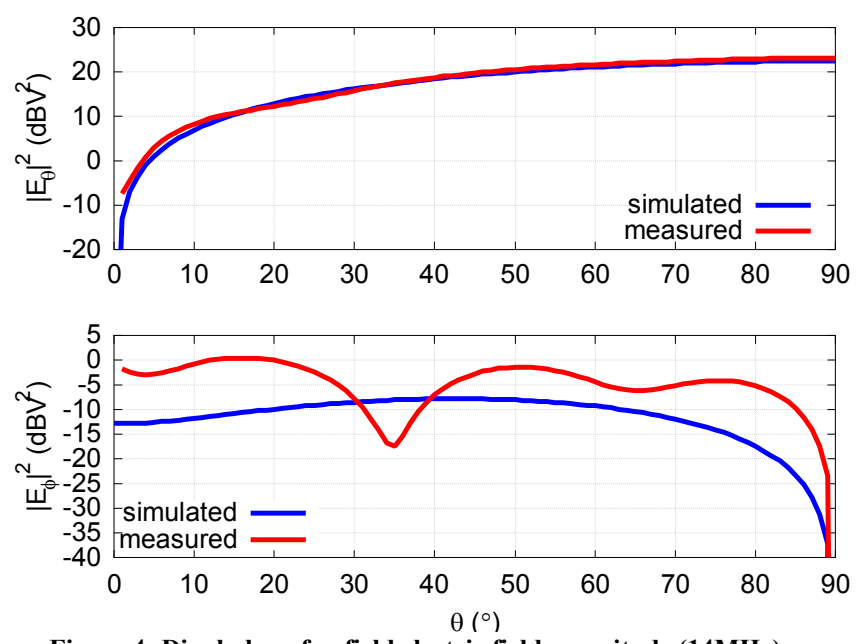

Figure 4: Dipole-loop far-field electric field magnitude (14MHz)

\section{CONCLUSION}

The extrapolated far-field antenna gain appears to be within $\pm 0.5 \mathrm{~dB}$ accuracy for the $\theta$-polarization. There is significantly more error for the $\varphi$-polarization, but this is likely a consequence low signal levels resulting in noisy $S$-parameter measurement. The low signal levels of the $\varphi$-polarization are a consequence of the conducting ground plane and the measured dipole-loop antenna being an inefficient $\varphi$-pol radiator in the HF band due to its electrically-small size.

[1] FEKO. (2016 Jan.) [Online]. Available: www.feko.info.

[2] C. Balanis, Advanced Engineering Electromagnetics, New York: Wiley \& Sons, 1989.

[3] T. Sarkar, P. Petre, A. Taaghol and R. Harrington, "An Alternative Spherical Near Field to Far Field Transformation," PIERs 16, pp. 269284, 1997 